\title{
Orbital compartment syndrome resulting in unilateral blindness in two dogs
}

\author{
Aurélie Sauvage ${ }^{1, *}$, Géraldine Bolen ${ }^{2}$, Sébastien Monclin ${ }^{1}$ and Magda Grauwels ${ }^{1}$ \\ ${ }^{1}$ Department of Clinical Sciences, Companion and Equine Animals, Ophthalmology, University of Liège, Quartier \\ Vallée 2, Avenue de Cureghem 3, 4000 Liège, Belgium \\ ${ }^{2}$ Department of Clinical Sciences, Companion and Equine Animals, Diagnostic Imaging, University of Liège, \\ Quartier Vallée 2, Avenue de Cureghem 3, 4000 Liège, Belgium
}

\begin{abstract}
Orbital compartment syndrome (OCS) is described in humans as an acute rise in intraorbital pressure following a severe and rapidly evolving orbital affection. It includes orbital oedema, haemorrhage or infection causing a marked reduction in local blood perfusion, and severely affecting the orbital neurovascular structures. If not promptly recognised and treated, it results in irreversible blindness. It is one of the rare ophthalmic surgical emergencies, requiring lateral canthotomy and cantholysis. This case report describes two canine cases of complex orbital, periorbital and facial abscesses resulting in OCS and permanent unilateral blindness diagnosed by ultrasonography, computed tomography and electroretinography.
\end{abstract}

Keywords: Dog, Globe tenting, Orbital abscess, Orbital compartment syndrome, Unilateral blindness.

\section{Introduction}

The differential diagnosis for acute unilateral blindness in dogs is substantial (Spiess and Pot, 2013; Garosi and Lowrie, 2014; Gould, 2018). It includes retrobulbar abscess, cellulitis and hematoma. Orbital abscess/cellulitis is common in dogs and has a fairly good prognosis (Rühli and Spiess, 1995; van der Woerdt, 2008; Kraijer-Huver et al., 2009; Oliver et al., 2009; Wang et al., 2009; Spiess and Pot, 2013; Vallefuoco et al., 2014; Betbeze, 2015; Tremolada et al., 2015; McDonald et al., 2016; Gould, 2018).

Only a few reports of orbital abscess/cellulitis as a cause of acquired unilateral blindness have been published in this species. The underlying pathophysiology has not been investigated. In some cases, optic nerve (ON) compression has been hypothesised (Tremolada et al., 2015; McDonald et al., 2016; Fisher et al., 2018).

In human medicine, orbital compartment syndrome (OCS) is an ocular emergency that can result in permanent $\mathrm{ON}$ blindness if not treated appropriately within two hours of the condition starting to develop. Clinical signs, which may present within minutes or hours, may include painful periorbital edema, peracute onset of visual loss, diploplia, and proptosis. It causes a rapid increase of the intraorbital pressure, which interferes with the blood perfusion of the orbital neurovascular structures and causes potentially irreversible ON damage.

Traumatic intraorbital bleeding and maxillo-facial surgeries are the main causes. Orbital compartment syndrome has also been described following fulminant orbital cellulitis/abscess and after spinal surgeries in prone position (Lima et al., 2009; Sun et al., 2014; Voss et al., 2016). In more than $75 \%$ of OCS cases "globe tenting", defined as a posterior globe angle of less than $130^{\circ}$, is reported and is considered a specific feature of increased intraorbital pressure (Dalley et al., 1989; Sun et al., 2014).

This case report describes two canine cases of complex orbital, periorbital and facial abscesses resulting in OCS and permanent unilateral blindness diagnosed by ultrasonography (US), computed tomography (CTscan), electroretinography (ERG).

\section{Case 1}

\section{Cases Details}

A four-year old English Staffordshire Bull-Terrier was presented as an emergency to the University of Liege Veterinary Emergency Service and Intensive Care Unit four days after a dog fight. The patient presented with peracute severe left periocular and facial swelling, dysorexia and lethargy (Fig. 1). Physical examination revealed pyrexia, left mandibular lymphadenomegaly, halitosis and pain on opening of the mouth. The ophthalmic examination showed severely swollen and warm eyelids, severe dorsotemporal exophthalmos with absence of globe retropulsion and eye movement, and two puncture wounds located ventrally to the left eye. Menace response, direct pupillary light reflex (PLR) and dazzle reflex were absent in the left eye. Slit lamp examination (SL-15, portable slit lamp, Kowa, Technop Belgium) showed a hyperaemic, swollen and protruding nictitating membrane, conjunctival chemosis, exposure ulcerative keratitis and mydriasis. 


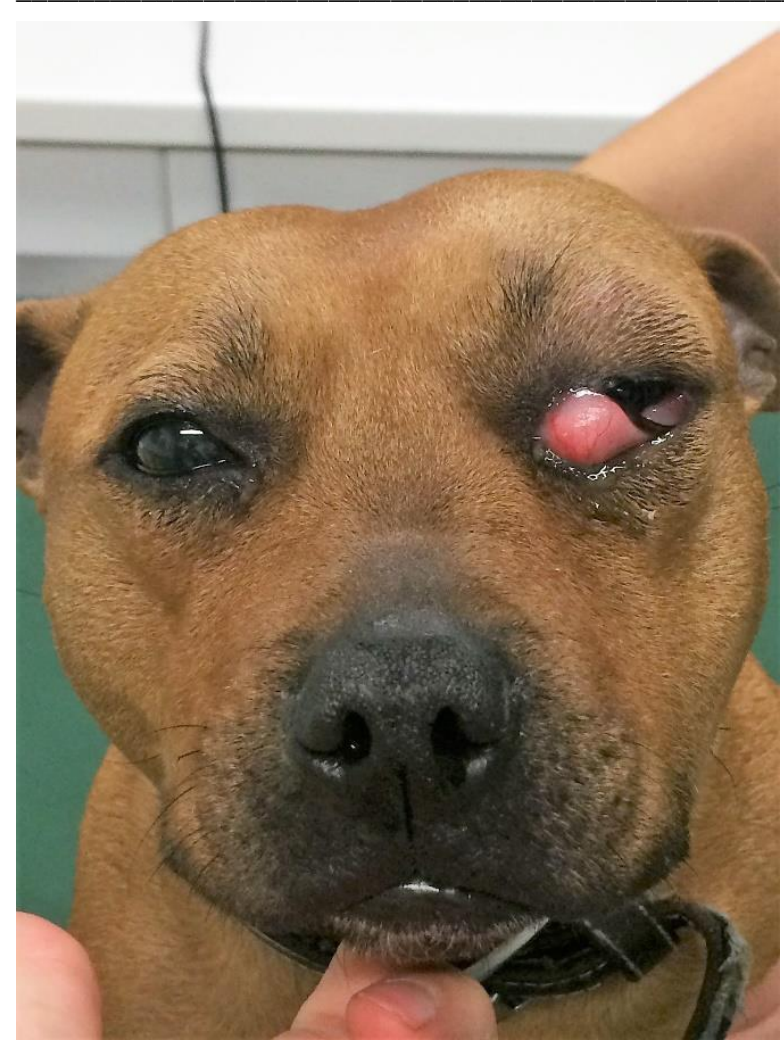

Fig. 1. Case 1 at presentation: hyperaemic, swollen and protrusion of the nictitating membrane, conjunctival chemosis, exophthalmos, periocular and periorbital swelling, left eye.

Indentation of the posterior wall of the globe was suspected by indirect ophthalmoscopy (Vantage Plus Indirect Ophthalmoscope, Keeler, Broomall, PA, USA), although the fundus could not be completely evaluated because of severe adnexal swelling. The right eye was unremarkable except for the absence of left to right indirect PLR. Intraocular pressure (IOP) was not measured on admission.

Ultrasound examination (Aloka Prosound SSDAlpha10, Aloka Co. Ltd., Tokyo, Japan equipped with a linear and a microconvex 5-10 $\mathrm{MHz}$ probes) of the left orbit showed a heterogeneous mass medial to the zygomatic arch, extending posteriorly and ventrally to the globe. Compression of the globe caused by the orbital mass was associated with marked globe tenting, identified as a conical deformation of the posterior wall of the globe (Fig. 2A). The mass was mainly hyperechoic with a hypoechoic region dorsally containing hyperechoic particles. Swelling and hypoechogenicity of the periocular subcutaneous soft tissues were also noticed. The left mandibular lymph node was enlarged with normal echogenicity and architecture.

Pre and post contrast CT scans (Siemens Somatom 16Slices, Germany) of the head were performed under general anaesthesia (midazolam $0.25 \mathrm{mg} / \mathrm{kg}$ and methadone $0.2 \mathrm{mg} / \mathrm{kg}$ premedication, propofol $2 \mathrm{mg} / \mathrm{kg}$ induction and isoflurane $+-2 \%$ maintenance), to further evaluate the origin and extension of the orbital mass, and to rule out a foreign body (Fig. $2 \mathrm{~B}-\mathrm{C}$ ). A $3 \times 3 \times 7 \mathrm{~cm}$ heterogeneous hypoattenuating $(25 \mathrm{HU})$ ovoid mass was observed temporally to the indented left globe and medial to the zygomatic arch. This mass extended medially to the ramus of the left mandible, up to and alongside the soft palate. The mass was poorly circumscribed by an unevenly thick irregular soft tissue-attenuating wall $(50 \mathrm{HU})$, which was contrastenhanced (70HU) and measured 2 to $5 \mathrm{~mm}$ in thickness. Dorsotemporal exophthalmia was present. No bony lesion or orbital foreign body was identified. Mild retropharyngeal and submandibular lymphadenomegaly was present. Zygomatic gland and peripheral soft tissues were enlarged. This large orbital and periorbital cavitary lesion with severe globe tenting was suggestive of an abscess, or less likely an orbital tumour with a necrotic component.

A fine needle aspiration was performed in the swollen area posteriorly to the last left upper molar tooth. A purulent discharge was aspirated and analysed. The cytological examination showed a marked suppurative inflammation with a mixed bacterial population, including intracellular filamentous bacteria within neutrophils. Staphylococcus pseudintermedius was identified in the discharge (aerobic and anaerobic culture, Synlab Veterinary, Belgium). The bacterium was sensitive to almost all antibiotics tested, including the prescribed medication amoxicillin-clavulanic acid and topical gentamycin gel (Table 1). A severe orbital and periorbital abscess was diagnosed.

Orbital drainage was performed promptly by two approaches. The oral mucosa was incised in the swollen area caudal to the last upper left molar. A blunt dissection with a delicate haemostat was performed in the direction of the orbit until the abscess was reached (Spiess and Pot, 2013; Gould, 2018). A large transconjunctival incision was performed in the ventral palpebral fornix. A large volume of purulent discharge flowed out. At the end of the procedure, a temporary partial tarsorrhaphy was performed in order to protect the cornea (Ethilon ${ }^{\circledR}$ 5-0).

Systemic medical treatment consisted of amoxicillinclavulanic acid $(20 \mathrm{mg} / \mathrm{kg}$ q $8 \mathrm{~h} \mathrm{IV}$ for 6 days then PO for 15 days) and metronidazole (20mg/kg q12h IV for 5 days). The latter was discontinued following culture and sensitivity results. Analgesia consisted of carprofen ( $2 \mathrm{mg} / \mathrm{kg}$ q12h IV for $48 \mathrm{~h}$ then PO for 7 days) and opioids (methadone $0.2 \mathrm{mg} / \mathrm{kg}$ q $4 \mathrm{~h}$ IV for $48 \mathrm{~h}$ followed by buprenorphine $0.015 \mathrm{mg} / \mathrm{kg}$ q6h IV for 3 days). Topical gentamycin gel and artificial tears (carbomer gel) were administered 6 times daily for 5 days, and then tapered until complete resolution of the corneal ulcer and other clinical signs. 

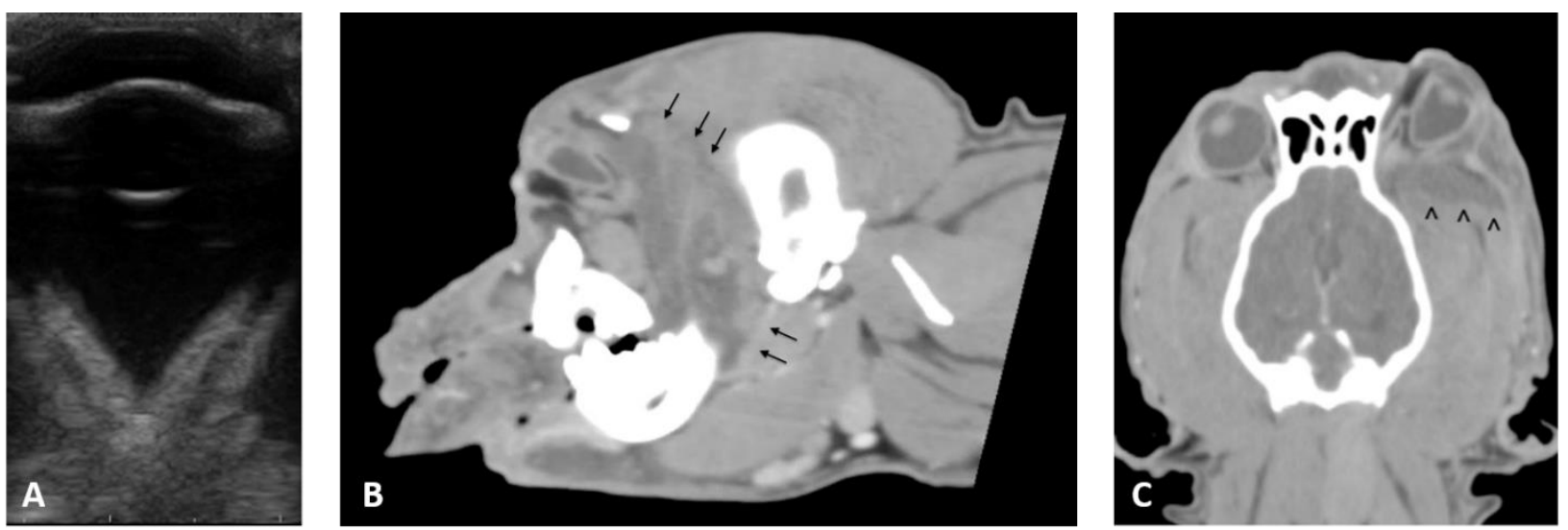

Fig. 2. Case 1 at presentation. (A): US parasagittal oblique image. (B and C): CT-scan dorsal and parasagittal reformatted post contrast images of the head, soft tissue window: conical shape of the left eye ("globe tenting") secondary to compression from a cavitary lesion containing septea surrounded by a not well circumscribed enhancing wall (arrows and arrowheads) with exophthalmos.

Left unilateral blindness and PLR abnormalities persisted throughout the postoperative period (checked daily for 5 days, at 3 weeks, at 2 months). The intraocular pressure remained within normal limits during the follow-up period.

A 3-week follow up CT scan revealed mild anterior displacement of the left globe with no further globe tenting, mild enlargement of the $\mathrm{ON}$ and near complete resolution of the cavitary lesion (Fig. 3).

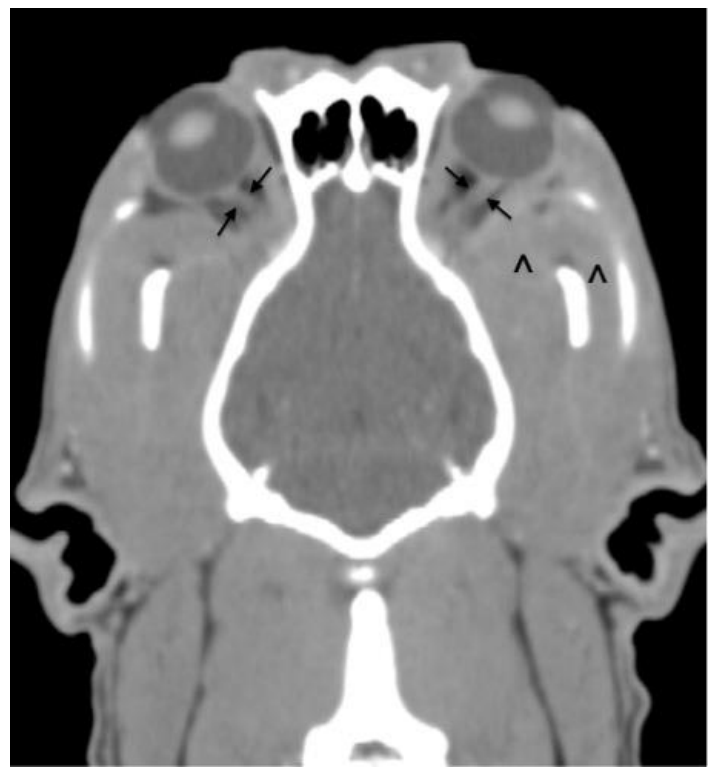

Fig. 3. Case 1: Three weeks after presentation, CT-scan dorsal reformatted post contrast images of the head, soft tissue window: globe shape returned to normal, almost complete resolution of the cavitary lesion with mild contrast enhancement of the left retro-orbital soft tissue (arrowheads). Arrows indicate the optic nerves. A mild enlargement on the left side can be appreciated ( $4 \mathrm{~mm}$ versus $3.4 \mathrm{~mm}$ on the right side).
Retropharyngeal and submandibular lymph nodes were decreased in size compared to the previous examination. Electroretinography (Dog Diagnostic Protocol - HMsERG, OcuScience, Henderson, Nevada, US) was performed on both eyes at 3 weeks and 2 months after initial presentation and was within normal limits (Ekesten et al., 2013).

Indirect ophthalmoscopy and fundus photography (Otomed Smartscope Vet2, Finland) (Fig. 4A-D) revealed optic disk atrophy at the final examination (Fig. 4D).

Further follow-ups were declined. The owner described during a telephone follow-up at 10 months after the initial admission, no further obvious eye problems, except for the persistence of left-sided blindness.

\section{Case 2}

A three-year old male Australian Shepherd crossbreed was referred to the University of Liege Ophthalmology unit for the management of a peracute large left orbital abscess, suspected by CT-scan performed by the referring veterinarian. Only the pre-contrast study was performed. It showed similarities with case 1. A large orbital and periorbital cavitary lesion extending ventrally within and around the medial pterygoid muscle was present. Globe tenting was also noted.

Physical examination revealed left mandibular lymphadenomegaly, pyrexia, and pain on opening of the mouth. Ophthalmic examination showed severe dorsolateral exophthalmos with absence of globe retropulsion, severely swollen and warm eyelids, as well as a large left-sided facial swelling (Fig. 5). Menace response and dazzle reflex were absent in the left eye. Slit lamp examination (SL-15, portable slit lamp, Kowa, Technop Belgium) showed a hyperaemic, swollen, ulcerated and protruding nictitating membrane, with severe conjunctival chemosis. 



Fig. 4. Case 1: (A): fundus left eye three weeks after presentation: peripheral focal striping, as result of previous indentation. Normal vessels. (B): fundus left eye two months after presentation: mild sign of optic disk atrophy. Normal vessels. (C and D): left optic disk three weeks (C) and two months (D) after presentation: optic disk atrophy progression.

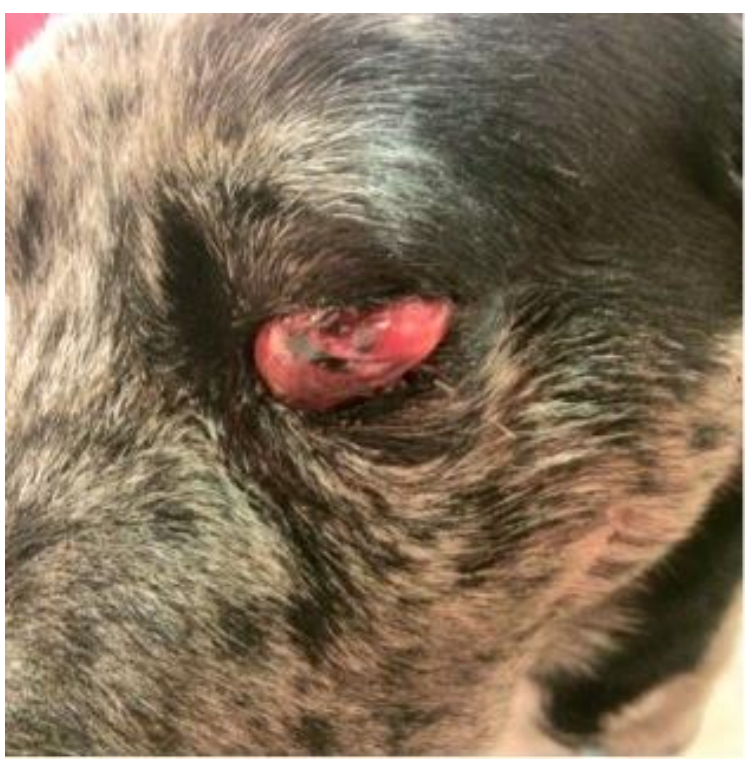

Fig. 5. Case 2 at presentation: hyperaemic, swollen and protrusion of the nictitating membrane, chemosis, exophthalmos, periocular and periorbital swelling, left eye.

Further ophthalmic examination was limited by the severe adnexal swelling, and IOP could not be measured. The right eye was unremarkable except for the absence of a left to right indirect PLR (slit lamp examination and indirect ophthalmoscopy (Vantage Plus Indirect Ophthalmoscope, Keeler, Broomall, PA, USA)). Oral orbital drainage was performed with the same technique described for case 1 . Two oral pockets of pus were also drained. Lateral canthotomy was performed with temporal transconjunctival drainage. A considerable quantity of purulent discharge was drained consistent with an abscess. Escherichia Coli was identified (aerobic and anaerobic culture, Synlab Veterinary, Belgium) and was sensitive to the initial antibiotic administered amoxicillin-clavulanic acid (20mg/kg q $8 \mathrm{~h} \mathrm{IV} \mathrm{for} 3$ days then PO for 15 days) (Table $2)$. Other treatment comprised of omeprazole $(1 \mathrm{mg} / \mathrm{kg}$ q24h IV for 3 days), meloxicam $(0.1 \mathrm{mg} / \mathrm{kg}$ q24h IV for 3 days then $\mathrm{PO}$ for 7 days) and opioids (buprenorphine $0.015 \mathrm{mg} / \mathrm{kg}$ q6h IV for 3 days then tramadol $2.5 \mathrm{mg} / \mathrm{kg}$ q12h PO for 3 days).
Table 1. Case 1 Antimicrobial susceptibility testing: Staphylococcus pseudintermedius.

\begin{tabular}{ll}
\hline Antibiotic & Sensitivity \\
\hline Benzylpénicilline & Resistant \\
\hline Ampicilline & Resistant \\
\hline Amoxicilline & Resistant \\
\hline Oxacilline & Sensitive \\
\hline Amoxicillin-clavulanic acid & Sensitive \\
\hline Cefalexine & Sensitive \\
\hline Cefovecine & Sensitive \\
\hline Gentamycine & Sensitive \\
\hline Erythromycine & Sensitive \\
\hline Clindamycine & Sensitive \\
\hline Oxytetracycline & Sensitive \\
\hline Marbofloxacine & Sensitive \\
\hline Enrofloxacine & Sensitive \\
\hline Pradofloxacine & Sensitive \\
\hline Sulfonamide Trimethoprim & Sensitive \\
\hline Mupirocine & Sensitive \\
\hline
\end{tabular}

Table 2. Case 2 Antimicrobial susceptibility testing: E. Coli.

\begin{tabular}{ll}
\hline Antibiotic & Sensitivity \\
\hline Ampicilline & Sensitive \\
\hline Amoxicilline & Sensitive \\
\hline Amoxicillin-clavulanic acid & Sensitive \\
Cefalexine & Sensitive \\
\hline Cefovecine & Sensitive \\
Gentamycine & Sensitive \\
\hline Erythromycine & Sensitive \\
\hline Clindamycine & Sensitive \\
\hline Oxytetracycline & Sensitive \\
Marbofloxacine & Sensitive \\
\hline Enrofloxacine & Sensitive \\
\hline Pradofloxacine & Sensitive \\
\hline Sulfonamide Trimethoprim & Sensitive \\
\hline Nitrofurantoine & Sensitive \\
\hline
\end{tabular}


Topical oxytetracycline ointment and artificial tears (carbomer gel) were administered 6 times daily for 3 days then tapered. Cold packs were applied every two hours for 10 minutes over 24 hours. The orbital abscess and exophthalmos resolved. Left-sided blindness and PLR abnormalities persisted throughout the postoperative follow-up period (checked daily for 3 days, then at 2, 4, 6 weeks and 4 months). Left optic disk atrophy progressed gradually at each follow-up visit. Fundic images (Optomed Smartscope Vet2, Finland) of both eyes were taken at 4 months. Severe atrophy of the left ON head as well as mild retinal vascular attenuation were seen (Fig. 6). The intraocular pressure remained within normal limits throughout the follow-up period. Owing to financial constraints, the owners declined a postoperative CT-scan and ERG.

\section{Discussion}

The diagnosis of OCS in human medicine relies on clinical presentation. Presenting signs include peracute deterioration in visual acuity, diplopia and PLR abnormalities (Soare et al., 2015; Broadway, 2016). Patients experience a rapid and painful increase in orbital pressure with severe swelling of the adnexae, tense lids, exophthalmia, reduced or absent eye movement, and globe tenting in $75 \%$ of cases (Lima et al., 2009; Sun et al., 2014; Soare et al., 2015; Voss et al., 2016).

In the above two case reports, very similar signs were noted: the severe facial swelling and exophthalmos occurred acutely and were associated with pain on opening of the mouth and globe tenting. In case 1 , the affected globe was immobile. The globe could not be examined in case 2. A menace response, direct left (affected globe) and left to right indirect PLRs were absent. Although the shape and position of the globes returned to normal following treatment, left-sided blindness and PLR abnormalities persisted throughout the follow-up period. These clinical findings were consistent with a prechiasmatic ON lesion and support a diagnosis of OCS. Differential diagnosis included glaucoma, primary retinal disease, direct damage and/or stretching of the ON and optic neuritis. The absence of fundus changes consistent with glaucoma and the maintenance of a normal IOP excluded glaucoma. In human medicine, the IOP is frequently increased in the acute phase of the condition. Measuring a decrease in IOP reflects the efficacy of the decompression treatment (Oester et al., 2012). In both cases, the IOP was not be measured on admission. In case 1 , it was omitted given the emergency. In case 2, there was no direct access to the cornea owing to the massive periocular cellulitis. Glaucoma and primary retinal disease could be ruled out in case 1 because of the normal ERGs at the 3- and 8-week follow-up visits. Secondary retinal atrophy developed following the ON disease in case 2.

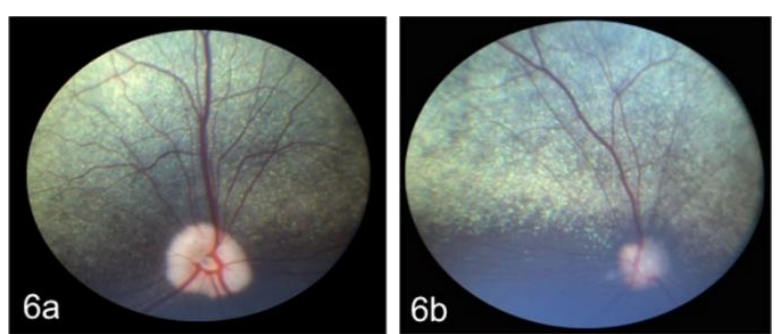

Fig. 6. Case 2, fundus 4 months after presentation. (a): right eye appears normal. (b): Left eye has a mildly attenuated vascular pattern and optic disk atrophy.

The anamnesis, the physical and ophthalmic examination permitted to exclude direct or iatrogenic ON damage. Since exophthalmia was moderate in both cases, we assumed that the moderate stretching of the $\mathrm{ON}$ was not responsible for the blindness. In the current literature, no information is available about how much the canine ON can be stretched before irreversible damage occurs. Retrobulbar optic neuritis could not be ruled out because neither MRI, nor visual evoked potentials or histopathology results were available. However, sufficient clinical and diagnostic tests supported OCS in the two cases presented here.

To the author's knowledge, this is the first description of OCS in veterinary literature.

Anatomical differences of the orbit, the globe and the ON vasculature between human and canine patients may explain a greater predisposition to OCS in people as compared to dogs. Orbital expansion possibilities in humans are minimal. The human orbit is a pyramid-like cavity limited by four bony walls containing the globe, lying on the orbital fat, with its neurovasculature structures. The central artery provides the main blood supply to the ON. The eye remains in place due to the extraocular muscles as well as the upper and lower eyelids, which are attached to the orbital rim by the medial and lateral canthal ligaments, and the inferior and superior septum. The ON has $8 \mathrm{~mm}$ of redundant length to accommodate eye movement (Voss et al., 2016).

Dogs have increased orbital expansion and facility to displace the globe due to an incomplete bony orbit and generally a more elongated skull. Furthermore, the ON is supplied by a complex network of arteries and veins, as compared to the central optic nerve artery and vein in humans.

The canine orbital ON blood supply is composed of the multiple short and two long posterior ciliary arteries and a number of inter-anastomosing vessels draining into the internal ophthalmic vein. The long posterior ciliary artery originates from the internal and external ophthalmic anastomosis (Miller, 2013; Murphy et al., 2013; Garosi and Lowrie, 2014). These anatomical differences may explain why OCS has not yet been described in dogs. 
Further studies are required to identify some predisposing factors within the canine species. Our hypothesis is that dolichocephalic dogs and/or dogs with normal lid lengths, which are tightly anchored to the orbital ligaments, would be more prone to OCS compared to brachycephalic dogs and/or dogs with macroblepharon, which allows greater orbital expansion and displacement of the globe.

Orbital compartment syndrome is an uncommon condition in human medicine but with dramatic sequelae and permanent vision loss if not promptly and appropriately managed. Early recognition of the clinical signs and surgical intervention for orbital decompression is required within 2 hours after the development of the specific symptoms in order to prevent blindness. Orbital decompression should not be delayed by any diagnostic work-up, such as diagnostic imaging. Surgical intervention consists mainly of lateral canthotomy and cantholysis, however in severe cases, an orbitotomy may be necessary to achieve adequate decompression of the orbit (Sun et al., 2014; Soare et al., 2015; Voss et al., 2016).

The dogs described here were referred more than 2 hours after the development of clinical signs and were already blind in the affected eye. Orbital drainage was performed late in the course of the disease and the affected eye remained blind during the follow-up period. The two dogs had complementary examinations before the surgical intervention. The time before development of blindness in canine OCS is unknown. In human medicine, the exact pathophysiology of OCS is unknown. The blindness may result from direct compressive effect on the $\mathrm{ON}$ axons, reduction in its blood supply leading to hypotensive and ischemic processes, or from a combination of the above processes (Voss et al., 2016).

Hypotensive and ischemic process of the $\mathrm{ON}$ is described as ischemic optic neuropathy (ION). It represents one of the major causes of blindness in human. It is characterised by ON hypoxia due to a reduction in local blood perfusion and pressure. Anterior- or posterior-ION are identified depending on the altered blood supply: the posterior ciliary artery or the central ON artery, respectively. Anterior-ION affects the ON head and posterior-ION the remaining ON.

In all types, ION develops after acute ischemia of the axons and causes axoplasmic flow stasis that rapidly results in swollen axons. Clinically, in cases of anteriorION, optic disk oedema appears in parallel with vision loss, and disk pallor develops after 2-3 weeks. In cases of posterior-ION, the optic disk and fundus appear initially normal, and optic disk pallor develops after 68 weeks (Hayreh, 2009).

In veterinary medicine, ION has been recently described by Mari et al. (2017) and Vinas et al. (2017).
Mari et al. (2017) presented a case of ION in a dog with acute bilateral blindness associated with primary systemic hypertension. They suspected posterior-ION given a normal funduscopy at admission and based on the typical MRI findings. Vinas et al. (2017) described a case of a dark red tubular mass arising from the $\mathrm{ON}$ head and confirmed the diagnosis of ION on histopathology.

We hypothesised that the canine OCS cases described here might suffer from posterior-ION as underlying pathophysiology. Optic disk oedema was not observed. Furthermore, in case 1, the ON was enlarged on the CT scan performed 3 weeks following the OCS, and ON head atrophy developed progressively over several weeks in both cases.

In veterinary medicine, apart from globe proptosis, there are very few publications on vision loss after acute orbital disease. In the study by McDonald et al. (2016), one of the three cases described suffering from a right orbital mucocele remained blind after a ventral transpalpebral anterior orbitotomy. The authors hypothesised a prechiasmal lesion associated with prolonged $\mathrm{ON}$ compression as the cause of the blindness. No ERG was performed to exclude the retinal contribution to the blindness. Fisher et al. (2018) described secondary blindness in 5 out of 33 cases evaluated for long-term ophthalmic complications following retrobulbar cellulitis/abscesses. No information on the possible causes were given. Griggs et al. (2016) described orbital and ocular disease in 6 dogs diagnosed with anticoagulant rodenticide toxicity. One of these patients showed a severe and acute unilateral orbital haemorrhage and remained blind. Vision loss here was attributed to ON compression secondary to orbital haemorrhage. We assume that the above-described cases could meet the clinical description of OCS, even if no information on globe tenting was given.

In conclusion, the clinical presentation, the diagnostic work-up and the clinical outcome allowed us to diagnose OCS in the two cases presented here.

In all cases of acute exophthalmia with severe orbital and periorbital swelling, whatever the cause, OCS has to be suspected and early recognition is essential, especially in dogs with tight small eyelid apertures. In order to save vision, very prompt diagnosis and very rapid decompression of the orbit should be performed prior to diagnostic testing. The diagnosis can be confirmed on ultrasound and/or computed tomography following orbital drainage.

\section{Acknowledgments}

The authors would like to thank the dogs' owners for their involvement in the follow-up examinations and treatments.

\section{Conflict of interest}

The authors declare that there is no conflict of interest. 


\section{References}

Betbeze, C. 2015. Management of orbital diseases. Top. Companion Anim. Med. 30, 107-117.

Broadway, D.C. 2016. How to test for a relative afferent pupillary defect (RAPD). Community Eye Health 29(96), 68-69.

Dalley, R.W., Robertson, W.D. and Rootman, J. 1989. Globe tenting: a sign of increased orbital tension. AJNR Am. J. Neuroradiol. 10(1), 181-186.

Ekesten, B., Komáromy, A.M., Ofri, R., PetersenJones, S.M. and Narfström, K. 2013. Guidelines for clinical electroretinography in the dog: 2012 update. Doc. Ophthalmol. 127(2), 79-87.

Fisher, M.C., Adrian, A.M., Demtriou, J., Nelissen, P. and Busse, C. 2018. Retrobulbar cellulitis and abscessation: focus on short- and long-term concurrent ophthalmic diseases in 41 dogs. J. Small Anim. Pract. doi: 10.1111/jsap.12924.

Garosi, L. and Lowrie, M. 2014. Neuroophthalmology. In BSVA Manual of Canine and Feline Ophthalmology, $3^{\text {rd }}$ edition, (ed. Gould, D. and McLellan, G.J.), British Small Animal Veterinary Association, pp: 357-383.

Gould, D. 2018. Diseases of the Orbit. In Slatter's Fundamentals of Veterinary Ophthalmology, $6^{\text {th }}$ edition, Elsevier, Inc., pp: 101-126.

Griggs, A.N., Allbaugh, R.A., Tofflemire, K.L., BenShlomo, G., Whitley, D. and Paulsen, M.E. 2016. Anticoagulant rodenticide toxicity in six dogs presenting for ocular disease. Vet. Ophthalmol. 19(1), 73-80.

Hayreh, S.S. 2009. Ischemic optic neuropathy. Prog. Retin. Eye Res. 28(1), 34-62.

Kraijer-Huver, I.M.G., ter Haar, G., DjajadiningratLaanen, S.C. and Boevé, M.H. 2009. Peri- and retrobulbar abscess caused by chronic otitis externa, media and interna in a dog. Vet. Rec. 165, 209-211.

Lima, V., Burt, B., Leibbovitch, I., Prabhakaran, V., Golberg, R.A. and Selva, D. 2009. Orbital compartment syndrome: the ophthalmic surgical emergency. Surv. Ophthalmol. 54(4), 441-449.

Mari, L., Stavinohova, R., Dominguez, E. , Premont, J., Oliver, J. and De Risio, L. 2017. Ischemic optic neuropathy in a dog with acute bilateral blindness and primary systemic hypertension. J. Vet. Intern. Med. 32(1), 423-427.

McDonald, J.E., Knollinger, A.M. and Dees, D.D. 2016. Ventral transpalpebral anterior orbitotomy: surgical description and report of three cases. Vet. Ophthalmol. 19(1), 81-89.

Miller, P. 2013. Orbit. In Slatter's Fundamentals of Veterinary Ophthalmology, $5^{\text {th }}$ edition, (ed. Maggs, D., Miller, P. and Ofri, R.) Elsevier Saunders, pp: 372-393.

Murphy, C.J, Samuelson, D.A. and Pollock, R.V.H. 2013. The eye. In Miller's Anatomy of the Dog, $4^{\text {th }}$ edition, (ed. Evans, H.E and de Lahunta, A.) Elsevier Saunders, pp: 746-785.

Oester, Jr., A.E., Fowler, B.T. and Fleming, J.C. 2012. Inferior orbital septum release compared with lateral canthotomy and cantholysis in the management of orbital compartment syndrome. Ophthalmic Plast. Reconstr. Surg. 28(1), 40-43. doi: 10.1097/IOP.0b013e31823646f3.

Oliver, J.A.C., Llabrès-Diaz, F.J., Gould, D.J. and Powell, R.M. 2009. Central nervous system infection with Staphylococcus intermedius secondary to retrobulbar abscessation in a dog. Vet. Ophthalmol. 12(5), 333-337.

Rühli, M.B. and Spiess, B.M. 1995. Retrobulbar spaceoccupying lesions in dogs and cat: symptoms and diagnosis. Tierarztl. Prax. 23(3), 306-312.

Soare, S., Foletti, J.M., Gallucci, A., Collet, C., Guyot, L. and Chossegros, C. 2015. Update on orbital decompression as emergency treatment of traumatic blindness. J. Craniomaxillofac. Surg. 43(7), 10001003.

Spiess, B.M. and Pot, S.A. 2013. Diseases and Surgery of the Canine Orbit. In Veterinary Ophthalmology, $5^{\text {th }}$ edition (ed. Gelatt KN), John Wiley \& Sons, Inc., pp: 793-831.

Sun, M.T., Chan, W.O. and Selva, D. 2014. Traumatic orbital compartment syndrome: importance of the lateral canthomy and cantholysis. Emerg. Med. Australas. 26, 274-278.

Tremolada, G., Milovancev, M., Culp, W.T.N. and Bleedorn, J.A. 2015. Surgical management of canine refractory retrobulbar abscesses: six cases. J. Small Anim. Pract. 56, 667-670.

Vallefuoco, R., Molas, C., Moissonnier, P. and Chahory, S. 2014. Lateral orbitotomy for treatment of an orbital abscess in a dog. J. Small Anim. Pract. 55, 531-534.

Van der Woerdt, A. 2008. Orbital inflammatory disease and pseudotumor in dogs and cats. Vet. Clin. North Am. Small Anim. Pract. 38, 389-401.

Vinas, M., D'Anna, C. and Giudice, C. 2017. Atypical clinical presentation, magnetic resonance imaging and histopathology of ischemic optic neuropathy (Poster). Abstracts: Annual Scientific Meeting of the European College of Veterinary Ophthalmologists, Estoril, Portugal May 18-21, 2017. Vet. Ophthalmol. 20(4), E12.

Voss, J.O., Hartwig, S., Doll, C., Hoffmeister, B., Raguse, J.D. and Adolphs, N. 2016. The "tight orbit": incidence and management of the orbital compartment syndrome. J. Craniomaxillofac. Surg. 44(8), 1008-1014.

Wang, A.L., Ledbetter, E.C. and Kern, T.J. 2009. Orbital abscess bacterial isolates and in vitro antimicrobial susceptibility patterns in dogs and cats. Vet. Ophthalmol. 12(2), 91-96. 